\title{
TECHNOLOGY
}

\section{Implantable loop recorder: evaluation of unexplained syncope}

\author{
R A Kenny, A D Krahn
}

Syncope is a relatively common occurrence in the general population accounting for up to $6 \%$ of hospital medical admissions and at least 3\% of accident and emergency visits each year. ${ }^{12}$ In a proportion of cases, syncope continues to pose a clinical dilemma despite the development of new diagnostic techniques. Mortality and serious morbidity associated with syncope are generally low overall but one year mortality is up to $33 \%$ in high risk groups of patients with a cardiac cause of symptoms. ${ }^{12}$ Recurrent syncope has additional deleterious effects on lifestyle, sense of physical wellbeing, and employment opportunities. ${ }^{3}$ Major obstacles to diagnosis are the intermittent nature of the symptoms, which are both periodic and often unpredictable, high spontaneous remission rates, and the lack of a diagnostic gold standard.

Despite the absence of a diagnostic gold standard for syncope and a paucity of data from randomised trials, several statements have emerged from a recent position paper on clinical guidelines from the American College of Physicians. ${ }^{4}$ In summary, these guidelines emphasise that:

- history, physical examination, and electrocardiography are the core of the investigations of syncope (with a combined diagnostic yield of $50 \%$ )

- neurological testing is rarely helpful unless additional neurological signs or symptoms are present (the diagnostic yield for electroencephalography, computed tomography, and Doppler ultrasound is only $6 \%$ )

- and patients are at higher risk of adverse outcomes if heart disease is known or suspected, or if patients have explained syncope. It is recommended that these patients should have additional cardiac testing including echocardiography, stress testing, head up tilt testing, Holter monitoring or intracardiac electrophysiological studies, either alone or in combination, depending on the clinical index of suspicion.

Despite these recommendations, syncope continues to pose diagnostic problems if it remains unexplained, occurs in the absence of structural heart disease, or if the clinical characteristics are atypical. Many centres use prolonged ambulatory monitoring as a first line of investigation, yet this most frequently identifies non-specific arrhythmias in the absence of symptoms. Documentation of significant arrhythmias or syncope during monitoring is rare. At best, symptoms correlating with significant arrhythmias occur in up to $4 \%$ of patients, asymptomatic arrhythmias occur in up to $13 \%$, and symptoms without arrhythmias in a further $17 \% .^{5-7}$ Patient activated external loop recorders have a higher diagnostic yield than prolonged ambulatory monitoring but do not yield a symptom-rhythm correlation in over two thirds of patients, either because of device malfunction, patient non-compliance, or inability to activate the recorder. ${ }^{89}$

Head up tilt testing reproduces presyncopal and syncopal symptoms in patients with vasovagal syncope. The specificity of head up tilt testing, in the absence of pharmacological provocation, is between $50 \%$ and $90 \%$, but test application is limited by variation in sensitivity, which ranges from $30 \%$ to $85 \% .^{10}{ }^{11}$ The diagnostic yield from cardiac electrophysiology ranges from $14-70 \%$; this variability is primarily dependent on the characteristics of patients studied, in particular the presence or absence of comorbid cardiovascular disease. ${ }^{12-16}$ Thus, the underlying cause of unexplained syncope is not determined in up to a quarter of patients despite investigations including head up tilt testing, ambulatory cardiac monitoring, external loop recording, and electrophysiological testing.

\section{Implantable loop recorder}

The implantable loop recorder, ILR (Reveal; Medtronic, Minneapolis, USA) is a more recent addition to diagnostic strategies for syncope. The ILR was developed to permit long term cardiac monitoring to capture the ECG during a spontaneous episode in patients without recurrence in a reasonable time frame. In most centres the ILR complements use of the external loop recorder. The device is generally implanted when an arrhythmia is suspected and when the external loop recorder has not yielded a diagnosis within a month. The device has the ability to "freeze" the current and preceding rhythm for up to 40 minutes after a spontaneous event and thus allows determination of the cause of syncope in most patients in whom symptoms are due to an arrhythmia. Using an activation device, the patient, family member, or friend "freezes" the loop during or after a spontaneous syncopal episode, storing 
the preceding segment, which is retrievable at a later stage. The ECG signal is stored in a circular buffer capable of retaining recorded rhythm. The device has a programmable memory bin selection for multiple events and real-time telemetry, and a shelf half life of at least 14 months. Both programming and interrogation can be performed with a standard pacemaker programmer. ${ }^{17-19}$

The insertion of the implantable loop recorder is straightforward, can be performed in a number of clinical settings, and numerous sites provide adequate sensing, including left pectoral, left submammary, right pectoral, and intercostal spaces. The device is $6.1 \times 1.9 \times 0.8 \mathrm{~cm}$, it weighs $17 \mathrm{~g}$, and has two bipolar sensing leads $3.7 \mathrm{~cm}$ apart within the shell of the device. The device is implanted under local anaesthetic via a small $(2 \mathrm{~cm})$ incision. The subcutaneous pocket is most frequently fashioned in the left pectoral region. The ILR is fastened to underlying tissues with non-absorbable sutures. The incision is then closed with absorbable sutures and a satisfactory electrogram verified after wound closure. Devices are explanted after a diagnosis is obtained.

The mean (SD) implant electrogram amplitude was 513 (276) $\mu \mathrm{V}$ and was stable during follow up. During prototype testing the amplitude of the sensed electrogram increased up to four months after implant. This may relate to bleeding and tissue oedema resulting from subcutaneous pocket formation, which gradually resolves over time as the pocket matures, resulting in an increase in the electrogram amplitude. ${ }^{19} 20$

In a series of 85 patients $^{19}$ the mean (SD) frequency of syncopal episodes 12 months before implant was 5.1 (5.5). Fifty two patients had had symptoms for longer than two years. Previous investigations including tilt table testing $(50 \%)$ and electrophysiological testing $(42 \%)$ had not provided a diagnosis. Only two patients had complications related to the implant; pocket infection in one and local pain in a second. Syncope or presyncope recurred in 53 patients $(62 \%)$ during the initial $5.3(2.1)$ months of follow up. Arrhythmias were present in 17 of 53 of patients, most of which were bradycardia. Six patients could not appropriately "freeze" after recurrent symptoms. The remaining 30 patients had sinus rhythm during symptoms, and continue to be followed to capture additional episodes. Only $20 \%$ of patients with sinus rhythm during symptoms had frank syncope, so that further follow up during actual syncope may provide a more accurate symptom-rhythm correlation.

\section{LIMITATIONS}

Limitations of the device include the inability to monitor blood pressure changes, the necessity for surgical implant, and a small risk of infection. Most patients in whom an arrhythmia is not documented during syncope have either hypotensive syndromes or psychogenic syncope. Hypotensive syndromes include vasovagal syncope, orthostatic hypotension, postprandial hypotension, and vasodepressor ca- rotid sinus hypersensitivity. A facility to track blood pressure behaviour in addition to heart rate behaviour during symptoms would undoubtedly advance real-time haemodynamic monitoring with implantable devices. The inability of the present device to detect hypotension is a limitation, although in some instances, hypotensive episodes can be inferred from patterns of heart rate behaviour before and during symptoms.

\section{ELDERLY PATIENTS}

The potential use of implantable loop recorders in older patients, for whom the prevalence rate of syncope is up to $10 \%$ per annum, has not been studied. Repeated outpatient investigations for many older adults are cumbersome and expensive. Health care and cost advantages of the implantable loop recorder in this population require further evaluation.

The cost of the device is half of that of a dual chamber pacing system. It might be argued that implantation of a pacemaker is preferable to that of a diagnostic device in older patients who have suspected but not confirmed bradyarrhythmias. However, clinical characteristics do not adequately predict diagnosis of bradyarrhythmias in a third of symptomatic patients who received an ILR: pacing is inappropriate in a third of patients and a further third who need pacing are missed (A Krahn, personal communication 1997).

\section{Conclusions}

In conclusion, the implantable loop recorder is a novel and useful diagnostic tool in patients with recurrent syncope of unknown cause. The device is associated with diagnostic sensed electrograms, is easy to implant, has a low adverse effect profile, and is of most benefit for establishing a diagnosis when symptoms are recurrent but too infrequent for conventional monitoring techniques. Further devices that capture blood pressure behaviour will enhance the diagnostic characterisation of syncope.

1 Day SC, Cook EF, Funkenstein H, et al. Evaluation and outcome of emergency room patients with transient loss of consciousness. Am f Med 1992;73:15-23.

2 Kapoor WN. Evaluation and outcome of patients with syncope. Medicine 1990;69:160-75.

3 Klein GJ, Gersh BJ. Yee R. Electrophysiological testing. The final court of appeal for diagnosis of syncope? Circulation 1995;92:1332-5.

4 Linzer M, Yang EH, Estes M, et al. Diagnosing syncope. Ann Intern Med 1997;126:989-96.

5 Gibson TC, Heitzman MK. Diagnostic efficacy of 24 hour electrocardiographic monitoring for syncope. Am f Cardiol

6 Clark PI, Glasser SO, Spoto E. Arrhythmias detected by ambulatory monitoring: lack of correlation with symptoms ambulatory monitoring: lack of correlation with

7 DiMarco P, Philbrick JT. Use of ambulatory electrocardiographic (Holter) monitoring. Ann Intern Med 1990;113:5368

8 Linzer M, Pritchett ELC, Pontiueu M, et al. Incremental diagnostic yield of loop electriocardiographic recorders in unexplained syncope. Am F Cardiol 1990;66:214-19.

9 Brown AD, Dawkins RD, Davies JG. Detection of arrhythmias: use of a patient-activated ambulatory electrocardiogram device with a solid state memory loop. Br Heart f 1989;58:251-3.

10 Kenny RA, Parry SW. Tilt table testings In: Malik M, ed. Clinical guide to autonomic tests. Boston/London: Kluwer Academic Publishers, 1998:67-100.

11 Sra JS, Anderson AJ, Sheikh SH, et al. Unexplained syncope evaluated by electrophysiologic studies and head-up tilt evaluated by electrophysiologic studies and

12 Denes P, Uretz E, Ezei MD, Borbola J. Clinical predictors of electrophysiological findings in patients with syncope of unknown origin. Arch Intern Med 1988;148:1922-8. 
13 Lacroix D, Dubuc M, Kus T, et al. Evaluation of arrhythmic causes of syncope: correlation between Holter monitoring, electrophysiological testing, and body surgace potentia mapping. Am Heart f 1991;122:1346-54

14 Bachinsky WB, Linzer M, Weld L, et al. Usefulness of clinical characteristics in predicting the outcome of electrophysiologic studies in unexplained syncope. Am f Cardiol 1992;69:1044-9.

15 Doherty JU, Pembrook-Rogers D, Grogan EW, et al. Electrophysiologic evaluation and follow-up. Characteristics of patients with recurrent unexplained syncope and prescynope. Am f Cardiol 1985;55:703-8.

16 Morady F, Shen E, Schwartz A, et al. Long term follow-up of patients with recurrent unexplained syncope evaluated by electrophysiologic testing. 7 Am Coll Cardiol 1983;2:1053-9.
17 Krahn AD, Klein GJ, Yee R. The etiology of syncope in patients with negative tilt table and electrophysiological testing. Circulation 1995;92:1819-24.

$18 \mathrm{Krahn}$ AD, Klein GJ, Yee R, et al. The etiology of syncope in patients with negative non-invasive and invasive testing: final results from a pilot study with an insertable loop recorder. Am $\mathcal{f}$ Cardiol. [In press.]

19 Krahn $A D$, Klein GJ, Yee R, et al. Implant experience with a new minimally invasive insertable loop recorder in 76 patients recurrent unexplained syncope. Circulation 1997; 96:2924-9.

20 Krahn AD, Klein GJ, Yee R, et al. Maturation of the sensed electrogram amplitude overtime in a new subcutaneous implantable loop recorder. PACE 1997;20:1686-90.

\section{IMAGES IN CARDIOLOGY}

\section{Recurrence of thymoma presenting as a superior vena cava syndrome}

A 60 year old woman was referred to our hospital complaining of swelling of the face. Seven years before admission she underwent a thoracotomy for thymoma. The resection of the tumour seemed to be complete, and no adjuvant treatment was given. Five months before admission she complained of oedema and intermittent flushing of the face. The clinical diagnosis was superior vena cava syndrome.

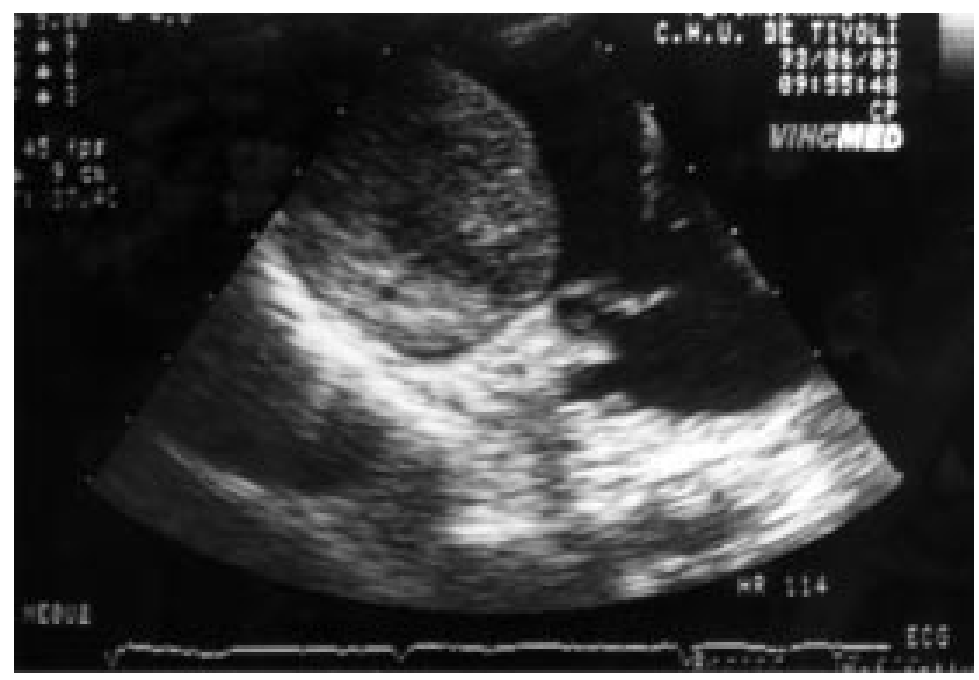

Computed tomography of the chest revealed no mediastinal adenopathy or parenchymal lesions, but rather thrombosis of the superior vena cava. Superior venacavography showed an enormous mass extending from the distal portion of the vena cava into the right atrium. Transthoracic echocardiography was negative, while transoesophageal echography showed a very large mass within the right atrium and superior vena cava.

Thoracotomy was difficult because of previous surgery. A tumour arising from the internal wall of the superior vena cava extending into the right atrium was removed with difficulty after incision of the atrium and vena cava. Pathology confirmed lymphoepithelial thymoma with spindle cells and moderate lymphocytic reaction.

This case seems to be the first description of thymoma recurrence within the superior vena cava and right atrium, seven years after the initial manifestation of thymoma; this underlines the long and insidious evolution of this tumour. It also demonstrates the usefulness of transoesophageal echocardiography in superior vena cava syndrome for detecting mediastinal or cardiac masses.

P LEFEBVRE A LELEUX C PHILIPPART 